PROCEEDINGS OF THE

AMERICAN MATHEMATICAL SOCIETY

Volume 138, Number 5, May 2010, Pages 1811-1819

S 0002-9939(10)10249-4

Article electronically published on January 6, 2010

\title{
INTEGRAL CONDITIONS ON THE SCHWARZIAN FOR CURVES TO BE SIMPLE OR UNKNOTTED
}

\author{
MARTIN CHUAQUI
}

(Communicated by Mario Bank)

\begin{abstract}
By considering integral bounds on the Schwarzian derivative we extend previous results on sufficient conditions for curves in euclidean spaces to be simple or unknotted. The conditions are optimal.
\end{abstract}

\section{INTRODUCTION}

Ahlfors' Schwarzian derivative for parametrized curves in $\mathbb{R}^{n}[\mathrm{Ah}]$ is a useful concept in the formulation of sufficient conditions for curves to be simple or unknotted ChG], Ch]. Recent results on the univalence of conformal parametrizations of minimal surfaces in $\mathbb{R}^{3}$ and holomorphic immersions of planar domains into $\mathbb{C}^{n}$ appeal to this operator in a crucial manner ChDO1, ChDO2. Injectivity in this context is ultimately a consequence of the disconjugacy of a linear equation of order two that involves a pointwise upper bound of the Schwarzian. The purpose of this paper is to establish new criteria of this type by considering instead integral bounds of the Schwarzian. In Theorems 1 and 2 below we deal with conditions that prevent a curve from self-intersecting or from becoming a knot. They are sharp in the sense that there exist parametrizations of non-simple or knotted curves that fail to satisfy the respective criterion by arbitrarily small amounts. The condition for simple curves involving a pointwise bound is satisfied by extremal parametrizations $f:(a, b) \rightarrow \mathbb{R}^{n}$ that reflect sharpness in the additional sense that $f([a, b])$ is closed. Corresponding extremal parametrizations for Theorem 1 fail to be smooth and exhibit a Schwarzian derivative equal to a delta function. Theorem 1 in this paper can be can be considered a kind of Schwarzian analogue of the result of Alexandrov and Reshetnyak (Theorem 5.8.1 in $[\mathrm{AR}$ ) that gives the sharp positive lower bound for the endpoint separation of a curve in euclidean space of total curvature less than $\pi$. It is interesting to note that the notion of total curvature requires very mild assumptions on the regularity of the curve, and that extremal behavior in their theorem is also manifested by a curvature function equal to a single delta in the midpoint.

Received by the editors November 24, 2008.

2000 Mathematics Subject Classification. Primary 53A04, 53A55; Secondary 34C10.

Key words and phrases. Ahlfors' Schwarzian, simple curves, knots, Möbius transformation.

This work was partially supported by Fondecyt Grant 1071019.

(C)2010 American Mathematical Society 


\section{AhlFors' Schwarzian}

Let $f:(a, b) \rightarrow \mathbb{R}^{n}$ be a $C^{3}$ curve with $f^{\prime} \neq 0$, and let $X \cdot Y$ stand for the euclidean inner product vectors $X, Y$ in $\mathbb{R}^{n}$ and $|X|^{2}=X \cdot X$. Ahlfors considered separately generalizations $S_{1} f$ and $S_{2} f$ of the real and imaginary parts of the usual Schwarzian in complex analysis $\mathrm{Ah}$. So far, only $S_{1} f$ has played a role for questions of injectivity. In $\mathrm{ChG}$ we showed that the real part of Ahlfors' Schwarzian, defined by

$$
S_{1} f=\frac{f^{\prime} \cdot f^{\prime \prime \prime}}{\left|f^{\prime}\right|^{2}}-3 \frac{\left(f^{\prime} \cdot f^{\prime \prime}\right)^{2}}{\left|f^{\prime}\right|^{4}}+\frac{3}{2} \frac{\left|f^{\prime \prime}\right|^{2}}{\left|f^{\prime}\right|^{2}},
$$

can be written in terms of the velocity $v=\left|f^{\prime}\right|$ and the curvature $k$ of the trace of $f$ as

$$
S_{1} f=\left(\frac{v^{\prime}}{v}\right)^{\prime}-\frac{1}{2}\left(\frac{v^{\prime}}{v}\right)^{2}+\frac{1}{2} v^{2} k^{2} .
$$

This expression is invariant under the Möbius transformations of $\mathbb{R}^{n} \cup\{\infty\}$. Observe that $S_{1} f=S s+\frac{1}{2} v^{2} k^{2}$, where $s=s(x)$ is arc length as a function of $x \in(a, b)$. Here, for a real-valued function $h$ with $h^{\prime} \neq 0, S h$ is the usual Schwarzian

$$
S h=\left(\frac{h^{\prime \prime}}{h^{\prime}}\right)^{\prime}-\frac{1}{2}\left(\frac{h^{\prime \prime}}{h^{\prime}}\right)^{2} .
$$

For a change of parameter $x=h(y)$,

$$
S_{1}(f \circ h)=\left[\left(S_{1} f\right) \circ h\right]\left(h^{\prime}\right)^{2}+S h .
$$

We recall the main results in $[\mathrm{ChG}], \mathrm{Ch}]$ :

Theorem A. Let $p=p(x)$ be a continuous real-valued function on an open interval $I$ such that any non-trivial solution of $u^{\prime \prime}+p u=0$ has at most one zero on I. Let $f: I \rightarrow \mathbb{R}^{n}$ be a $C^{3}$ curve with $f^{\prime} \neq 0$. If $S_{1} f \leq 2 p$, then $f$ is one-to-one on $I$ and admits a spherically continuous extension to the closed interval, which is also one-to-one unless the trace of $f$ is a circle, in which case $S_{1} f \equiv 2 p$.

Theorem B. Let $f:[-1,1) \rightarrow \mathbb{R}^{3}$ parametrize a simple closed curve in $\mathbb{R}^{3}$. If the periodic continuation of $f$ is $C^{3}$ and $S_{1} f(x) \leq 2 \pi^{2}$ for all $x \in(-1,1)$, then $f([-1,1))$ is unknotted.

When formulating Theorem B on an interval $[a, b)$, the resulting upper bound after the linear change of parameter becomes $8 \pi^{2} /(b-a)^{2}$. Let $l(I)$ be the length of an interval $I$ and let ${ }^{+}$denote positive part.

Theorem 1. Let I be an open interval, and let $f: I \rightarrow \mathbb{R}^{n}$ a $C^{3}$ curve with $f^{\prime} \neq 0$. If

$$
\int_{I}\left(S_{1} f\right)^{+}(x) d x \leq \frac{8}{l(I)},
$$

then $f$ admits a spherically continuous extension to the closed interval $\bar{I}$ and $f(\bar{I})$ is simple. 
Theorem 2. Let $J=[a, b)$, and let $f: J \rightarrow \mathbb{R}^{3}$ parametrize a simple closed curve in $\mathbb{R}^{3}$. If the periodic continuation of $f$ is $C^{3}$ and

$$
\int_{J}\left(S_{1} f\right)^{+}(x) d x \leq \frac{32}{l(J)}
$$

then $f(J)$ is unknotted.

In Theorem 1, for any $\epsilon>0$ there is a parametrization $f$ with $\int\left(S_{1} f\right)^{+} d x<$ $8 / l(I)+\epsilon$ for which $f(I)$ is not simple, and similarly for Theorem 2. Examples will be provided in the last section.

\section{Proofs}

Let $p(x) \geq 0$ and $u(x)$ be a solution of $u^{\prime \prime}+p u=0$ with initial conditions, say at $x=0, u(0)=0, u^{\prime}(0)=1$. If $u(x) \geq 0$ for $0 \leq x \leq x_{2}$, then $u(x) \leq x$ because it is concave. By integrating twice we see that for such $x$

(5) $u(x)=x-\int_{0}^{x}(x-t) p(t) u(t) d t \geq x-\int_{0}^{x} t(x-t) p(t) d t \geq x-\frac{x^{2}}{4} \int_{0}^{x} p(t) d t$.

The following result is well known (see, e.g., [ㅍ. Corollary 5.1]). For the convenience of the reader we include a brief proof.

Lemma 1. Let $p=p(x)$ be continuous on $(a, b)$, and suppose the equation

$$
u^{\prime \prime}+p u=0
$$

admits a non-trivial solution with two zeros in $(a, b)$. Then

$$
\int_{a}^{b} p^{+}(x) d x>\frac{4}{b-a} .
$$

Proof. Suppose $u \not \equiv 0$ is a solution of (6) with consecutive zeros $a<x_{1}<x_{2}<b$. Because $p \leq p^{+}$, the Sturm comparison theorem guarantees the existence of a nontrivial solution of the equation $u^{\prime \prime}+p^{+} u=0$ that vanishes twice. In other words, we may assume that the coefficient in (6) is non-negative. Also, it is sufficient to limit attention to the case in which $x_{1}=0$ and $u^{\prime}\left(x_{1}\right)=1$. For $x \in J=\left(0, x_{2}\right)$ we have from (5) that

$$
u(x) \geq x-\frac{A}{4} x^{2}
$$

where $A=\int_{0}^{x_{2}} p(x) d x$. We have used that $t(x-t) \leq x^{2} / 4$. Therefore,

$$
\frac{A}{4} x_{2}^{2}-x_{2} \geq-u\left(x_{2}\right)=0,
$$

which implies that

$$
\int_{a}^{b} p(x) d x \geq A=\int_{0}^{x_{2}} p(x) d x \geq \frac{4}{x_{2}}>\frac{4}{b-a} .
$$

The proof shows that the extremal situation occurs when the entire mass of $p$ is concentrated exactly at the midpoint between both zeros. Indeed, by letting $p$ be a delta function at the origin, one obtains zeros at \pm 2 for the solution $u(x)=2-|x|$ of (6) (in the appropriate weak sense). 
Proof of Theorem 1. Without loss of generality we may assume that $a=-b$ and that $\left|f^{\prime}(0)\right|=1$. Because the analysis of extension is symmetric at both endpoints, it is enough to show the extension on $(0, b]$. Let $p=f(0)$ and consider the curve $\phi=T \circ f$ for the Möbius transformation

$$
T(q)=\frac{q-p}{|q-p|^{2}} .
$$

Then $S_{1} \phi=S_{1} f$, and it was shown in [ChG] that the function

$$
u=\left|\phi^{\prime}\right|^{-1 / 2}=\frac{|f-p|}{\sqrt{\left|f^{\prime}\right|}}
$$

satisfies

and therefore also

$$
u^{\prime \prime}+\frac{1}{2}\left(S_{1} f\right) u \geq 0,
$$

$$
u^{\prime \prime}+\frac{1}{2}\left(S_{1} f\right)^{+} u \geq 0
$$

whenever $f \neq p$. The initial conditions are $u(0)=0$ and $u^{\prime}(0)=1$. On $[0, b)$ consider the solution $w$ of

$$
w^{\prime \prime}+\frac{1}{2}\left(S_{1} f\right)^{+} w=0, \quad w(0)=0, w^{\prime}(0)=1 .
$$

It follows from (5) that for $x \in(0, b)$

$$
w(x) \geq x-\frac{x^{2}}{2 b}>0,
$$

with the same inequality holding for $u(x)$ by the Sturm comparison. We conclude that for $x \geq b / 2$,

and hence

$$
u(x) \geq \frac{3 b}{2},
$$

$$
\int_{b / 2}^{b} u^{-2}(x) d x<\infty .
$$

Therefore $\phi((b / 2, b))$ has finite length, which shows that $\phi$ admits a continuous extension to $x=b$.

If $f(\bar{I})$ is not simple, then $f(\alpha)=f(\beta)=p$ for some $a \leq \alpha<\beta \leq b$. We may choose $\beta-\alpha$ smallest possible so that $f$ is injective on $(\alpha, \beta)$. Note that

$$
\int_{\alpha}^{\beta}\left(S_{1} f\right)^{+} d x \leq \int_{a}^{b}\left(S_{1} f\right)^{+} d x \leq \frac{8}{b-a} \leq \frac{8}{\beta-\alpha},
$$

which means that, after relabeling, we may assume that $a=\alpha$ and $b=\beta$. Consider the composition $\psi=T \circ f$ with the Möbius inversion

$$
T(q)=\frac{q-p}{|q-p|^{2}} .
$$

Then $S_{1} \psi=S_{1} f$ and $\psi(a)=\psi(b)=\infty$. Again $u=\left|\psi^{\prime}\right|^{-1 / 2}$ satisfies

$$
u^{\prime \prime}+\frac{1}{2}\left(S_{1} f\right)^{+} u \geq 0 \text {. }
$$

Fix $c \in(a, b)$ and let $w$ be the solution on $(a, b)$ of

$$
w^{\prime \prime}+\frac{1}{2}\left(S_{1} f\right)^{+} w=0, \quad w(c)=u(c), w^{\prime}(c)=u^{\prime}(c) .
$$


Lemma 1 shows that $w$ cannot have two zeros on $(a, b)$, and thus simple arguments based on the convexity or concavity of $w$ show that it has well-defined limits at both endpoints $a, b$. If $w(d)=0$ at some interior point, say $d<c$, then the argument presented in the previous paragraph shows that $w(x)>0$ for $x \in(d, b)$ and $w(x) \geq \rho>0$ near $x=b$. By Sturm comparison, $u(x) \geq w(x)$ on $(c, b)$, and thus

$$
\int_{c}^{b} u^{-2}(x) d x<\infty
$$

contradicting that $\psi(b)=\infty$.

Suppose, on the other hand, that $w(x)>0$ on $(a, b)$. If $w(a)>0$, then $\psi(a)$ is finite, a contradiction. If $w(a)=0$, we see from (5) that, near $x=b, w(x)$ will be bounded below by some positive constant because $\left(S_{1} f\right)^{+}$is a continuous function. Again we reach a contradiction. This proves the theorem.

Proof of Theorem 2. The proof follows that of Theorem B. The key step is to show that if $\Gamma=f([a, b])$ is a knot, then it can be laid out to form a planar, closed, nonsimple curve for which the real part of the Schwarzian has not increased. This procedure is based on the results of Brickell and Hsiung $[\mathrm{BH}]$ in the course of their proof of the Fary-Milnor theorem.

For $p \in \mathbb{R}^{3}$, let $C_{p}$ denote the shell of $\Gamma$ with vertex $p$ defined as the (developable) surface consisting of all segments $[p, q]$ with $q \neq p$ on $\Gamma$. The indicatrix $I_{p}$ of $C_{p}$ is the curve on the sphere $\mathbb{S}^{2}$ traced by the vectors $(q-p) /|q-p|$; its length $l\left(I_{p}\right)$ is called the total angle of $I_{p}$. A key result established in $[\mathrm{BH}]$ is that $\Gamma$ is unknotted if $l\left(I_{p}\right)<3 \pi$ for all $p \in \Gamma$ (see also Ch] ). Therefore, if $\Gamma$ is a knot, then there is a point $p \in \Gamma$ for which $l\left(I_{p}\right) \geq 3 \pi$. The cases $l\left(I_{p}\right)>3 \pi$ and $l\left(I_{p}\right)=3 \pi$ must be dealt with separately. Suppose first that $l\left(I_{p}\right)>3 \pi$. As the point $p$ moves to a point $p^{\prime}$ slightly away from $\Gamma$, the number $l\left(I_{p^{\prime}}\right)$ varies continuously, except for a jump increment in $\pi$. It follows that there exists $p^{\prime} \notin \Gamma$ for which $l\left(I_{p^{\prime}}\right)>4 \pi$. Since $l\left(I_{r}\right)$ is a continuous function of $r \in \mathbb{R}^{3} \backslash \Gamma$ and since $l\left(I_{r}\right) \rightarrow 0$ as $|r| \rightarrow \infty$, there exists $p_{0} \notin \Gamma$ for which $l\left(I_{p_{0}}\right)=4 \pi$. As was shown in [Ch], the shell $C_{p_{0}}$ can be laid out isometrically onto the plane in a way that $\Gamma$ traces out a $C^{3}$ closed curve $\gamma$ with winding number 2 with respect to the point in the plane corresponding to the vertex $p_{0}$.

In the case $l\left(I_{p}\right)=3 \pi$ we lay out $\Gamma$ as before, with $p$ corresponding in the plane to, say, the origin. A variant of the argument principle allowing for zeros on the curve (see, e.g., [N, p. 131]) implies that $\gamma$ cannot be simple: the point $0 \in \gamma$ contributes $\pi$ to the total variation of argument and therefore $\gamma$ must in addition wind around the origin once.

In either case, let $g:[a, b) \rightarrow \mathbb{R}^{2}$ be the induced parametrization of $\gamma$ defined on the original interval of definition of $f$. Since the laying out procedure preserves arc length, then $v_{g}=\left|g^{\prime}\right|=\left|f^{\prime}\right|=v_{f}$. Also, the curvature of $\gamma$ is equal to the curvature of $\Gamma$ relative to the surface $C_{p_{0}}$ and therefore not larger than the curvature of $\Gamma$. We conclude from (11) that $S_{1} g \leq S_{1} f$. Since $\gamma$ is not simple, it can be subdivided into closed curves $\gamma_{1}, \gamma_{2}$ which are differentiable except at the point where $\gamma$ has self-intersection (see Figure 1). 

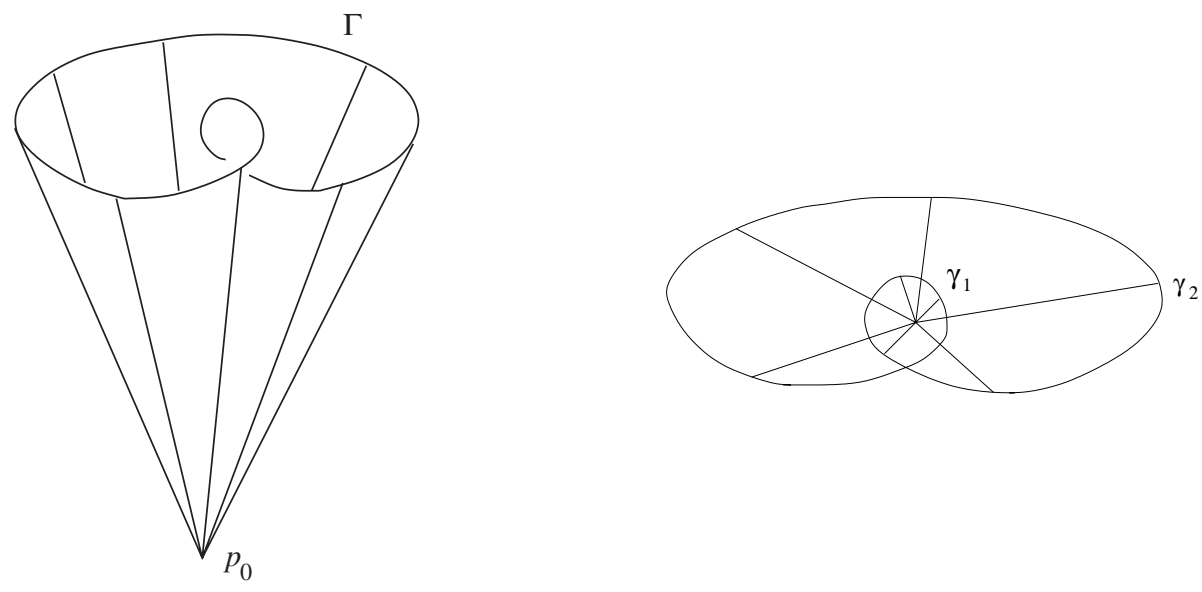

FiguRE 1

Because $g$ is periodic, one can find intervals $I_{1}=\left[x_{1}, x_{2}\right], I_{2}=\left[x_{3}, x_{4}\right]$ of total length $b-a$ such that

(i) $g_{1}=\left.g\right|_{I_{1}}:\left[x_{1}, x_{2}\right] \rightarrow \gamma_{1}$ and $g_{2}=\left.g\right|_{I_{2}}:\left[x_{3}, x_{4}\right] \rightarrow \gamma_{2}$;

(ii) the parametrizations $g_{1}, g_{2}$ are $C^{3}$ on the open subintervals.

Because $g_{1}, g_{2}$ are closed, it follows from Theorem 1 that

$$
\int_{x_{1}}^{x_{2}}\left(S_{1} g_{1}\right)^{+} d x>\frac{8}{x_{2}-x_{1}}, \quad \int_{x_{3}}^{x_{4}}\left(S_{2} g_{2}\right)^{+} d x>\frac{8}{x_{4}-x_{3}} .
$$

But

therefore

$$
\frac{1}{x_{2}-x_{1}}+\frac{1}{x_{4}-x_{3}} \geq \frac{4}{\left(x_{2}-x_{1}\right)+\left(x_{4}-x_{3}\right)}=\frac{4}{b-a}
$$

a contradiction.

$$
\int_{a}^{b}\left(S_{1} f\right)^{+} d x \geq \int_{a}^{b}\left(S_{1} g\right)^{+} d x>\frac{32}{b-a},
$$

\section{EXAMPLES}

Example 1. Let $\epsilon>0$ and fix $I=(-1,1)$. We seek a non-injective $f: I \rightarrow \mathbb{R}^{n}$ for which

$$
\int_{I}\left(S_{1} f\right)^{+}(x) d x<4+\epsilon
$$

Let $p_{n}$ be a sequence of positive, even, $C^{\infty}$ functions with $\int_{I} p_{n} d x=2+\epsilon / 4$ converging to $p_{0}=(2+\epsilon / 4) \delta_{0}(x)$ in the sense that

$$
\lim _{n \rightarrow \infty} \int_{I} p_{n} h d x=\int_{I} p_{0} h d x
$$

for all $h$ continuous on $I$. For $c=1 /(1+(\epsilon / 8))<1, u_{0}(x)=c-|x|$ is a solution of $u^{\prime \prime}+p_{0} u=0$ with zeros at $\pm c$. By writing the differential equation $u^{\prime \prime}+p_{n} u=0$ in integral form, it is easy to see that, for $n$ sufficiently large, an even solution $u_{n}$ of $u^{\prime \prime}+p_{n} u=0$ will have exactly two zeros on $I$, say at $\pm x_{n}$. For such an $n$ let

$$
g(x)=\int_{0}^{x} u_{n}^{-2}(t) d t .
$$


This function is odd, increasing and $g\left(x_{n}\right)=-g\left(-x_{n}\right)=\infty$; the intervals $g\left(\left(x_{n}-\delta, x_{n}+\delta\right)\right)$ and $g\left(\left(-x_{n}-\delta,-x_{n}+\delta\right)\right)$ overlap. We can avoid the point at infinity by taking $f=T \circ g$ for a suitable Möbius transformation that maps $\mathbb{R} \cup\{\infty\}$ onto a circle in the plane. Again we have an overlap along some arc of the circle. The Schwarzian $S_{1} f=S_{1} g=S g$ is left invariant, and a standard calculation shows that $S g=2 p_{n}$. Because

$$
2 \int_{I} p_{0}(x) d x=4+\frac{\epsilon}{2}
$$

we see that for $n$ sufficiently large,

$$
\int_{I}\left(S_{1} f\right)^{+}(x) d x<4+\epsilon,
$$

as desired.

Example 2. Fix $J=[-1,3)$ and $\epsilon>0$. We seek $f: J \rightarrow \mathbb{R}^{3}$ with a $C^{3}$ periodic continuation for which $f(J)$ is a knot and

$$
\int_{J}\left(S_{1} f\right)^{+} d x<8+\epsilon .
$$

The first step is the construction of a $C^{3}$ periodic parametrization $g: J \rightarrow \mathbb{R}^{2}$ satisfying (12) for which $g(J)$ goes around a circle twice. On each half $J_{1}=[-1,1)$ and $J_{2}=[1,3)$, the parametrization $g$ will trace out the circle once in identical fashion. We give the details for $J_{1}$. Let $p_{0}(x)$ be a smooth, non-negative, even approximation of $4 \delta(x)$ with support in a small neighborhood of $x=0$, for which

$$
4<\int_{J_{1}} p_{0}(x) d x<4+\frac{\epsilon}{4}
$$

Consider the solution $u_{0}$ of

$$
u_{0}^{\prime \prime}+p_{0} u_{0}=0, \quad u_{0}(0)=1, u_{0}^{\prime}(0)=0 .
$$

Because the optimal constant in (3) for the interval $J_{1}$ is 8 , we see from (13) that by taking $p$ sufficiently close to the delta function, the function $u_{0}$ will have zeros at points $\pm x_{0} \in J_{1}$ close to the endpoints of $J_{1}$. Note that $u_{0}$ is linear away from the support of $p$. Consider

$$
F(x)=\int_{1}^{x} u^{-2}(t) d t .
$$

Then $F\left(\left(-x_{0}, x_{0}\right)\right)=\mathbb{R}$ and $F\left(-x_{0}\right)=F\left(x_{0}\right)=\infty$, so that some Möbius transformation $G=T \circ F$ will map $\left[-x_{0}, x_{0}\right)$ onto a circle. One can adjust the coefficient $p$ so that $u_{0}=0$ at exactly \pm 1 . But a linear decay to 0 will prevent the continuation of $G$ to $J_{2}$ to be $C^{3}$ at $x=1$. To see this, suppose $u_{0}(x)=\alpha(1-x)$ near $x=1$, and let

$$
G=\frac{1}{F} .
$$

Then $\left|G^{\prime}\right|=F^{\prime} / F^{2}$ is an even function with non-vanishing derivative at $x=1$ and therefore cannot be continued to $J_{2}$ as a smooth periodic function. This shows that we need to modify the asymptotic of $u$ at the zeros in a way that the resulting 
function $\left|G^{\prime}\right|$ has a vanishing derivative there. For example, near $x=1$ one can take

$$
u(x)=\frac{(1-x)-\frac{1}{3}\left(1-x^{2}\right)^{3}}{\sqrt{1-2 x\left(1-x^{2}\right)^{2}}}
$$

which gives near 1

$$
F(x)=\frac{1}{(1-x)-\frac{1}{3}\left(1-x^{2}\right)^{3}} .
$$

The resulting function $\left|G^{\prime}\right|$ now has vanishing derivative at $x=1$. A straightforward calculation shows that $u^{\prime \prime}+q u=0$ for a function $q$ that is negative. This means that we can modify the original approximation $p_{0}$ to an even function $p$ with

$$
\int_{J_{1}} p^{+}(x) d x<4+\frac{\epsilon}{4}
$$

for which the even solution of $u^{\prime \prime}+p u=0$ vanishes exactly at \pm 1 in accordance with (14). A Möbius transformation of $F$ has the desired property and can now be extended periodically to $J_{2}$ as a smooth parametrization $g$ that goes around a circle $C$ twice. Note that $S_{1} g=S_{1} F=2 p$; hence

$$
\int_{J}\left(S_{1} g\right)^{+}(x) d x<8+\frac{\epsilon}{2} .
$$

By slightly modifying the curvature and with a small cost in $\int_{J}\left(S_{1} g\right)^{+}(x) d x$, we can arrange that the curve traced out by $g$ restricted to $J_{2}$ be almost a circle, disjoint from $C$ except for the point of tangency $g(1)$.

In the final step, we modify $g$ to a parametrization $f$ for which the trace is knotted, once more with negligible cost in $\int_{J}\left(S_{1} g\right)^{+}(x) d x$. This can be accomplished by replacing a small portion of one of the arcs at the point of tangency by a very thin tubular neighborhood, along which the new arc of $f$ will knot around once. This procedure introduces torsion, on which the Schwarzian is independent. It is easy to see that both the modified curvature and velocity remain arbitrarily close to their original values as long as the tubular neighborhood is thin enough. This finishes the example.

\section{REFERENCES}

[Ah] L.V. Ahlfors, Cross-ratios and Schwarzian derivatives in $\mathbb{R}^{n}$, Complex Analysis, 1-15, Birkhäuser, Basel, 1988. MR981397 (90a:30055)

[AR] A.D. Alexandrov and Yu.G. Reshetnyak, General theory of irregular curves, Kluwer Academic, Dordrecht, 1989. MR.1117220 (92h:53003)

[BH] F. Brickell and C.C. Hsiung, The total curvature of closed curves in Riemannian manifolds, J. Diff. Geom. 9 (1974), 177-193. MR0339032 (49:3795)

[Ch] M. Chuaqui, On Ahlfors' Schwarzian derivative and knots, Pacific J. Math. 231 (2007), 51-62. MR2304621 (2008m:53003)

[ChG] M. Chuaqui and J. Gevirtz, Simple curves in $\mathbb{R}^{n}$ and Ahlfors' Schwarzian derivative, Proc. Amer. Math. Soc. 132 (2004), 223-230. MR2021266 (2005f:53001)

[ChDO1] M. Chuaqui, P. Duren and B. Osgood, Univalence criteria for lifts of harmonic mappings to minimal surfaces, J. Geometric Analysis 17 (2007), 49-74. MR2302873 (2008d:31001)

[ChDO2] M. Chuaqui, P. Duren and B. Osgood, Injectivity criteria for holomorphic curves in $\mathbb{C}^{n}$, Pure and Applied Mathematics Quarterly 7 (2011), no. 1, 215-243. 
[H] P. Hartman, Ordinary differential equations, $2^{\text {nd }}$ Edition, Birkhäuser, Boston, MA, 1982. MR658490 (83e:34002)

[N] Z. Nehari, Conformal mapping, Dover Books on Advanced Mathematics, Dover Publ. Inc., New York, 1975. MR0377031 (51:13206)

Facultad de Matemáticas, Pontificia Universidad Católica de Chile (PUC), Avenida Vicuña Mackenna 4860, Santiago, Chile

E-mail address: mchuaqui@mat.puc.cl 